\title{
AUTONOMOUS LUGGAGE CARRIER HEXAPOD
}

\author{
Vaibhav Malhotra \\ Department of Computer Engineering \\ Thapar Institute of Engineering and Technology, Patiala, Punjab, India
}

\begin{abstract}
In the last few years, there has been a lot of advancements in Robotics and Computer Science, to an extent that Robotic Devices are now included in our daily life and help us in our day to day activities. The more important thing is that this is not the peak, this is just the beginning, there is a lot of research going on in this field with the aim of making our quality of life better. This paper presents a similar project undertaken to help elderly people in carrying their luggage around the airport terminal. This paper presents the methodology and algorithms used to develop the device. The prototype has been tested in open spaces, while simulations have been done to analyze its scalability to accommodate multiple robots coexisting.
\end{abstract}

Keywords- Raspberry Pi, Robotics, Hexapod, Luggage Carrier, Automation, Technology, Hexapod Robot

\section{INTRODUCTION}

Recent advancements in technology and communication have caused a revolution in all fields. Now, the challenge is to find a way to incorporate technology in our daily lives to such an extent that it feels natural and enhances our quality of life.

One of the fields that has seen the most advancement is robotics and automation. Robot devices are aimed not only at assisting people, instead of replacing them, but also at adopting the role of companions. Therefore, they must be designed to interact with untrained people, and a great effort must be made to enable users to accept them easily.

Such robots have to work in untrained environments like homes, offices, Airports (in this case), etc. They have to take into account all the random events that can occur and all the random objects they can encounter and prepare for them. In this case, the robot needs to move about the airport terminal, therefore, it needs to consider people walking by, various obstacles, and all of the random situations it may encounter. These types of problems are dealt with using the latest cutting-edge sensors and motion planning algorithms.

One of the most tiring aspects of flights is the carrying of the luggage through long terminals, especially for aged people, and everyone else in general. This paper presents a model that is in this domain and uses the latest research and developments to make the process of carrying luggage in airport terminal easier. Although this project was taken up with the intention of helping elderly people carry their luggage, it is clear that this system can help everyone at the airport and make their travel easier and faster.
If charged on a pay-per-use basis, this system also has great potential for generating significant income to airport authorities which can be used in the maintenance and advancement of airport facilities.

\section{COMPONENTS}

1) 12 DOF Hexapod chassis -

This is the most commonly used hexapod chassis used by students for research purposes because of its durability and ease of control which will help in this project to a great extent.

2) Raspberry $\mathrm{Pi}-$

Raspberry Pi is the name of a series of singleboard computers made by the Raspberry Pi Foundation, a UK charity that aims to educate people in computing and create easier access to computing education. The Raspberry Pi is a very cheap computer that runs Linux, but it also provides a set of GPIO (general purpose input/output) pins that allow you to control electronic components for physical computing and explore the Internet of Things (IoT). Any version of raspberry $\mathrm{Pi}$ would suffice as long as it has wireless connectivity otherwise a Wi-Fi module must be used.

3) Pi-Cam -

The Pi camera module is a portable lightweight camera that supports Raspberry Pi. It communicates with Pi using the MIPI camera serial interface protocol. It is normally used in image processing, machine learning, or surveillance projects. This will be used for person detection and obstacle avoidance. In this project, we use three 5 MP Pi-Cams.

4) LiPo Battery(11.1 V) -

A lithium polymer battery, or more correctly lithium-ion polymer battery (abbreviated as LiPo, LIP, Li-poly, lithium-poly, and others), is a rechargeable battery of lithium-ion technology using a polymer electrolyte instead of a liquid electrolyte. High conductivity semisolid (gel) polymers form this electrolyte. These batteries provide higher specific energy than other lithium battery types and are used in applications where weight is a critical feature which is 
also true for our project. The more mAh battery we use the longer will be the runtime of the Carrier between charge.

5) The belt for person Recognition -

This is a self-designed belt for recognition of the person. It will have a colored pattern for recognition by the camera of the Carrier and a pattern at the center for easy identification by the object detector (As Shown in Fig. 1). This will be worn by the person and on the back, a colored pattern will be present. The colored pattern will serve two purposes: -

a. Recognition of the person to follow among the crowd.

Every Carrier will have a specific belt assigned. When the person wears the camera and comes in view of the camera the Carrier detects the belt and follows it, thus following the person wearing it.

b. Preventing false positives when many Carriers work together.

Every belt has a different pattern and every Carrier is assigned a specific belt, this prevents Carrier from getting confused on whom to follow if two people wearing a belt come into the field of view of the camera. The Carrier will identify the belt associated with it and follow the respective person.

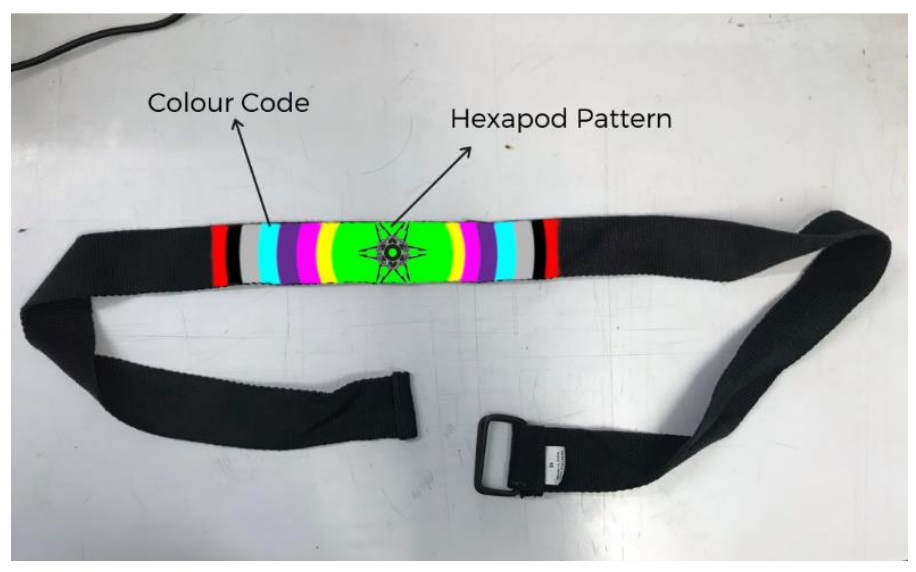

Fig. 1 - Belt to be worn by the person

6) LCD battery indicator with a speaker for low battery warning -

Simple LCD which shows battery remaining, estimated time before next charge, and has a buzzer associated with it for low battery warning. After the buzzer reserve battery is used and the Carrier can only be functional for around 10 minutes depending upon the load.
7) Carrier for Baggage -

A carrier upon the hexapod chassis for carrying the luggage.
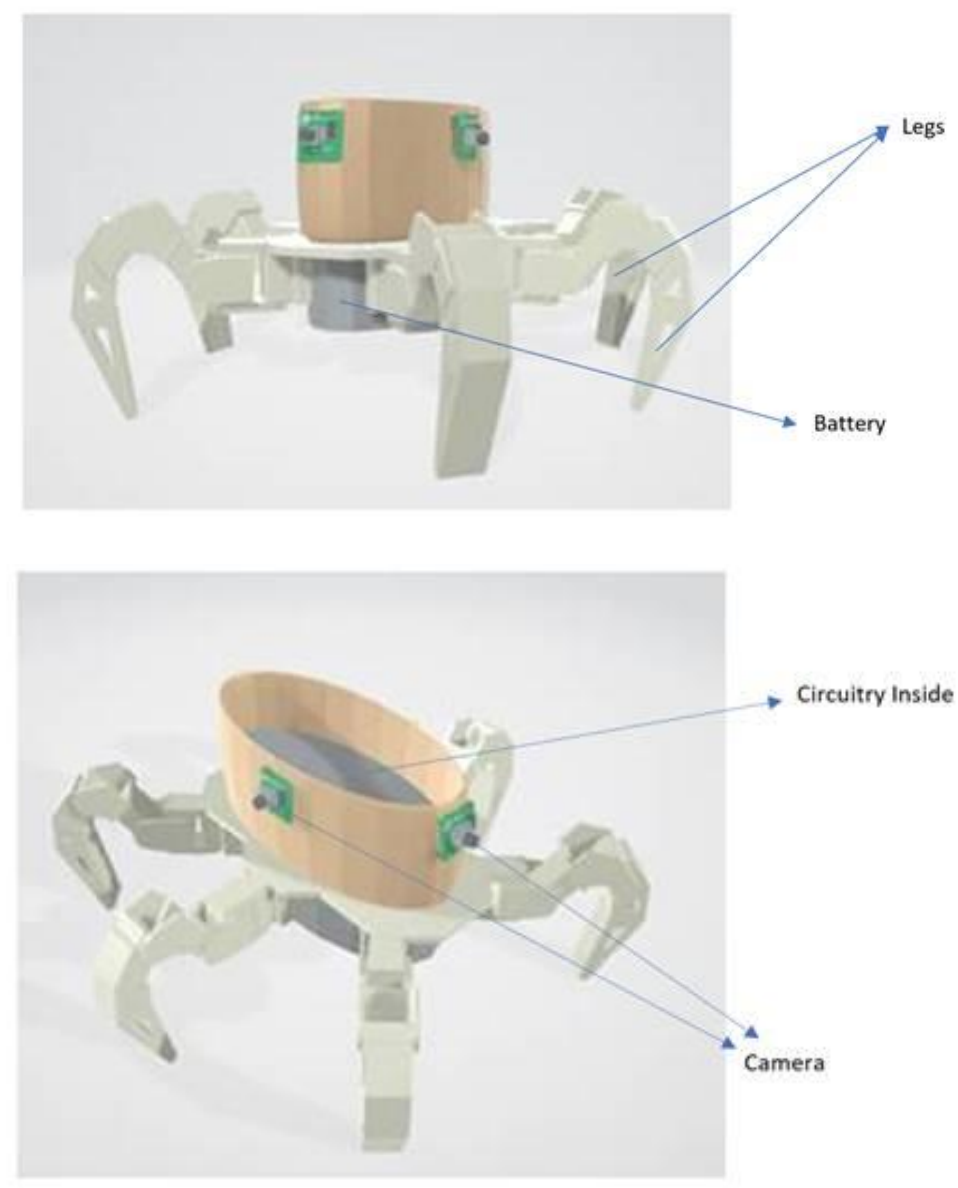

Fig. $2 \& 3-3 D$ Device Model

\section{PROPOSED MODEL}

\section{A. ALGORITHM}

Each Carrier would have a QR code for its identification. When the person turns the main switch on a welcome message will be displayed on the LCD Display, followed by a message to connect it to a device. The procedure to be followed by the user is as follows: -

- Turn on the device through the main switch on the Carrier.

- Scan the QR code sticker on the Carrier through your Mobile App.

- Wear the belt ensuring that the color code of the belt is not covered by any piece of clothing like a jacket, etc

- $\quad$ See the camera view in your app and wait for the Carrier to detect your belt (by standing in front of the camera) which will be indicated by a beep sound from the Carrier and a message on your mobile App.

- $\quad$ Place all your luggage in the Carrier. 
- Press start on your App, your Carrier will stand up and start following you.

- Now you can walk anywhere and the Carrier will follow you. You can continuously see the camera feed from the Carrier on your phone app and you will also get controller buttons on the app if you want to control the Carrier.

- If at any point the Carrier loses the view of your belt or fails to detect it, it will automatically stop, giving you an alert on your phone, which will prompt you to calibrate the device again.

- When you reach your destination, you can press finish on your phone, and the Carrier will go back in crouch position enabling you to pick up your luggage with ease.

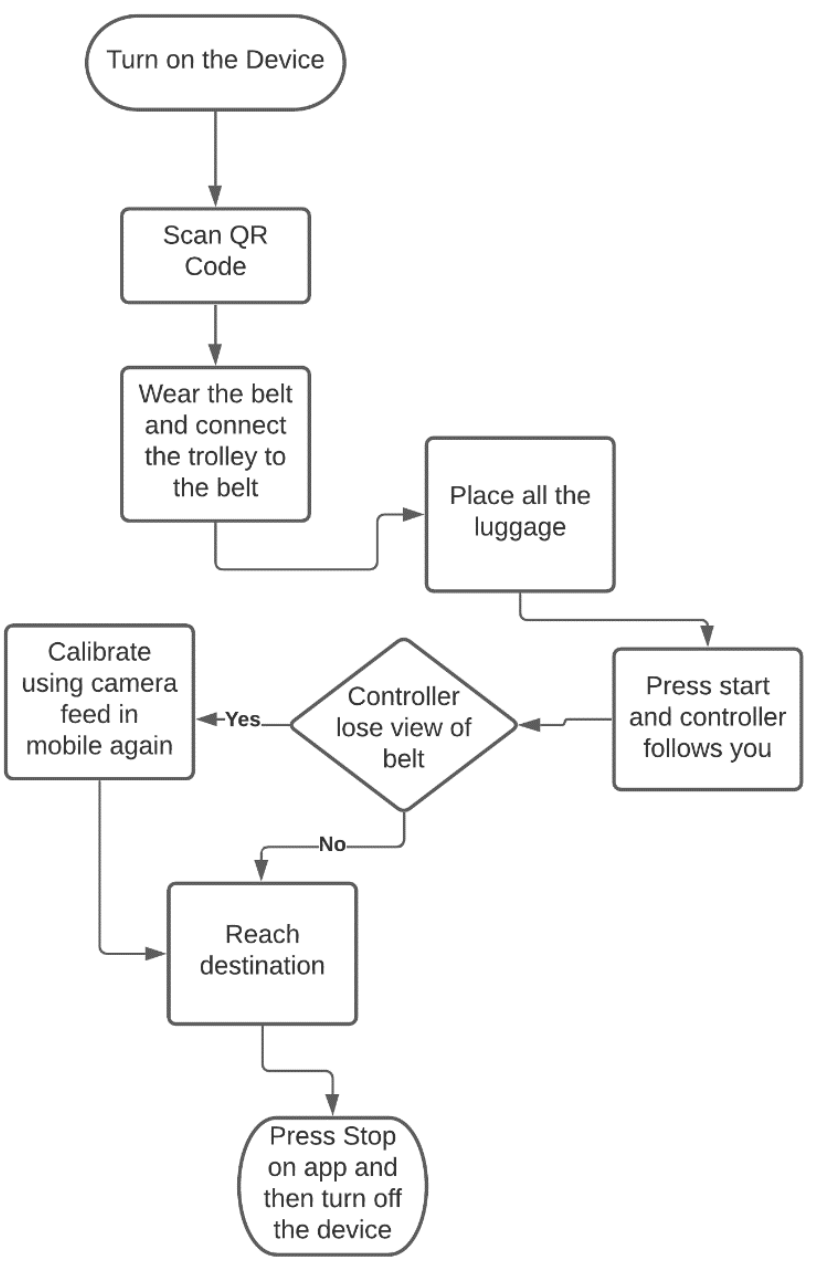

Fig. 4 - Flowchart for Autonomous Luggage Carrier

\section{B. WORKING}

When a person turns on the Carrier, it prompts the person to scan the QR code with his/her mobile. The QR code contains the static IP address allocated to the Carrier. The mobile device sends a request to the respective IP address and connects with the Carrier. The mobile and Carrier need to be on the same WiFi network(Airport WIFI).
Now the person will be able to see the live feed from Carrier Camera on their Mobile Device.

Next, the person needs to wear the belt and stand in front of the camera till it detects the belt. When the Raspberry Pi detects the belt it will assign a marker to the center of the belt and will show that marker on the camera feed in your device. The Carrier will also sound a beep to signal that it is ready and will give notification on your app for the same.

The Raspberry Pi uses YOLO Algorithm to detect the belt, its details will be explained later in this paper. The Carrier will try to keep the marker at the center of the

camera view and at a particular distance from the camera, thus following the belt and hence the person wearing it

\section{WALKING GAIT}

There are a lot of options for walking gait for hexapods. In this experiment, we use $3+3$ gait, which is the most common gait used for regular walking on a smooth surface.[1][2]

In this gait, the hexapod legs are divided into two groups, labeled L1, L2, L3 for the left side and R1, R2, R3 for the right side

(Fig. 5). The legs are grouped into two groups (L1, L3, R2, and L2, R1, R3). Locomotion is depicted through the foothold diagram.

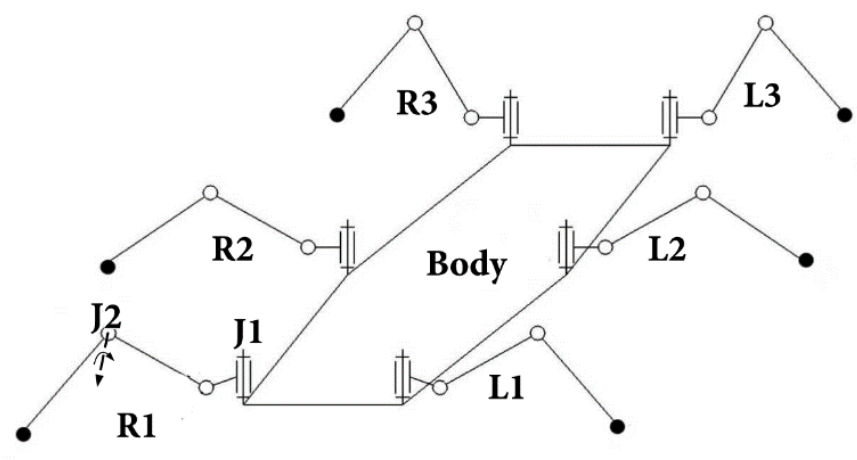

Fig. 5 - Hexapod Diagram

In the foothold pattern diagram, white bars represent the swing phase of a leg and the black bars represent the stance phase. In this gait, 3 legs always stay on the ground for support, and 3 legs swing and move the hexapod in the desired motion. (Fig. 6) 


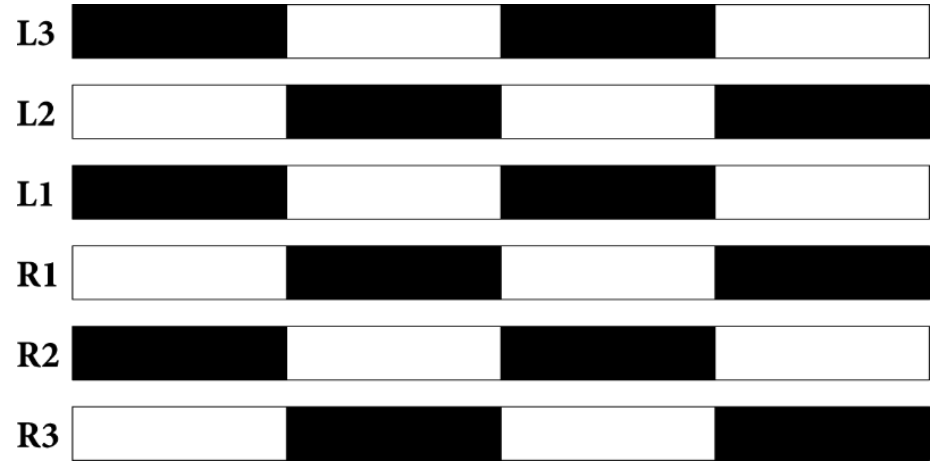

Fig. 6 - Foothold Pattern for ' $3+3$ ' Regular Gait

The most important advantage of this gait is that it provides speed and stability even with luggage on the Carrier. Since 3 legs are always on the ground, the center of gravity of the Carrier will never shift to any extreme position which could result in instability and Carrier falling.

\section{APP INTERFACE}

The Carrier will be wirelessly connected to the user's phone app by scanning the QR code on the Carrier. Through the app, the user will be able to see the live feed from all the cameras in the Carrier. Moreover, the user can control some parameters of the Carrier related to the person following functionality utilizing the controls provided in the app.

The user will be able to view the live camera feed from the Carrier cam, they will be able to turn off auto-following mode and control the Carrier by the use of control buttons provided on the app. They will be able to turn the Carrier on and off to put remove luggage. This will allow the user to tune the way he/she wants the Carrier to perform. Moreover, by using this interface, the user can know other robot parameters, like the battery status and so forth.

\section{THE CODE}

There are two main components of the code that can be confusing and need explanation.

\section{A. BELT DETECTION}

Yolo algorithm [3] is used to train an Object Detector to find the belt from the camera view and mark a point on the center of the belt and keep following that point.

The YOLO version used for this device is YOLO v3 - Tiny [4], which is an improved version, and this is used due to its less computing power requirement, and improvements in speed and accuracy.

The detector is trained on 1000 images (different angles and lighting conditions) for the belt.
After all the belts are detected in the view by detecting the hexapod pattern, the program moves outward from there and looks for the specific colors pattern assigned to the particular Carrier in all the bounding boxes, and once it finds that, a marker is assigned to the center point of the respective bounding box.

If no box matches the pattern allocated, or no belt is recognized in the view of the camera, a notification is sent to the User's app notifying them that the Carrier has lost contact with the belt and asking them to use the camera view and realign the belt to the Carrier.

\section{B. MOVEMENT}

To follow the person, a simple algorithm is used. Once a marker is available and the person presses start on his/her app, the Carrier stands up, and then it tries to keep the marker point, in the center of the camera, this takes care of making turns following the person.

To keep the distance between the person and Carrier constant we make use of the bounding box.

When a bounding box is finalized to be followed, its breadth (How many pixels are taken up by the box horizontally) is noted, to ensure ease of calculation and smoothening of movement, the percentage of area occupied by the box horizontally is calculated. Then the Carrier tries to maneuver to keep the percentage constant, thus following the belt and hence the person. If the percentage decreases the carrier moves forward and vice-versa.

When a person presses stop on his/her app, all the bounding box information is erased and the Carrier goes in crouch position which helps the person in picking up their luggage.

\section{CONCLUSION}

For most people, the most tiring factor to consider while traveling through an airport is to carry all their luggage through long terminals. The system proposed in this paper is aimed to help elderly people and everyone else in general in carrying their luggage through the airport and making their trips more comfortable and easier. It will save time and energy of the travelers.

\section{FUTURE SCOPE}

There is a lot of scope of improvement and advancement in this project. There are a lot of features that can be added in this project.

One such feature that will be added in the future is Path planning using SLAM. The robot can be integrated with ROS and SLAM algorithms to make it more robust using state of the art motion planning algorithms in the future. This will make the user experience even easier and help the Carrier to avoid obstacles by mapping the whole Airport terminal and then planning its path automatically avoiding all the static obstacles using onboard computational power. 


\section{International Journal of Engineering Applied Sciences and Technology, 2020 \\ Vol. 5, Issue 7, ISSN No. 2455-2143, Pages 102-106 \\ Published Online November 2020 in IJEAST (http://www.ijeast.com)}

\section{REFERENCES}

[1] Chang, Q, \& Mei, F, (2018). A Bioinspired Gait Transition Model for a Hexapod Robot, Journal of Robotics, vol. 2018, Article ID 2913636, 11 pages. https://doi.org/10.1155/2018/2913636

[2] Ding, X \& Wang, Z \& Rovetta, A \& Zhu, J.M.. (2010). Locomotion Analysis of Hexapod Robot. 10.5772/8822.

[3] Redmon, J., Divvala, S., Girshick, R., \&amp; Farhadi, A. (2016). You Only Look Once: Unified, Real-Time Object Detection. 2016 IEEE Conference on Computer Vision and Pattern Recognition (CVPR). doi:10.1109/cvpr.2016.91

[4] Adarsh, P., Rathi, P., \& Kumar, M. (2020). YOLO v3Tiny: Object Detection and Recognition using one stage improved model. 2020 6th International Conference on Advanced Computing and Communication Systems (ICACCS). doi:10.1109/icaccs48705.2020.9074315

[5] Lokanath, M., \&amp; Sai, G. A. (2017). Live video monitoring robot controlled by web over internet. IOP Conference Series: Materials Science and Engineering, 263, 052027. doi:10.1088/1757-899x/263/5/052027

[6] Du, Juan. (2018). Understanding of Object Detection Based on CNN Family and YOLO. Journal of Physics: Conference Series. 1004. 012029. 10.1088/1742$6596 / 1004 / 1 / 012029$

[7] Russakovsky, O., Deng, J., Su, H., Krause, J., Satheesh, S., Ma, S., . . Fei-Fei, L. (2015). ImageNet Large Scale Visual Recognition Challenge. International Journal of Computer Vision, 115(3), 211-252. doi:10.1007/s11263-015-0816-y

[8] Zak, M., \& Rozman, J. (2015). Design, construction and control of hexapod walking robot. 2015 IEEE 13th International Scientific Conference on Informatics, 302307.

[9] Hwang, M., Huang, C., \& Liu, F. (2018). A Hexapod Robot with Non-Collocated Actuators. Applied System Innovation, 1(3), 20. doi:10.3390/asi1030020

[10] Chaudhari, H., \& Gadge, S.S. (2015). Raspberry Pi Technology: A Review. 